“드 2014 IEEE. Personal use of this material is permitted. Permission from IEEE must be obtained for all other uses, in any current or future media, including reprinting/republishing this material for advertising or promotional purposes, creating new collective works, for resale or redistribution to servers or lists, or reuse of any copyrighted component of this work in other works." 


\section{Improving Dental Care Recommendation Systems using Trust and Social Networks}

\author{
Sojen Pradhan, Valerie Gay \\ Faculty of Engineering and IT \\ University of Technology Sydney (UTS) \\ Sydney, Australia \\ Sojendra.Pradhan@uts.edu.au
}

\author{
Surya Nepal \\ ICT Centre \\ Commonwealth Scientific and Industrial \\ Research Organisation (CSIRO) \\ Marsfield, Australia
}

\begin{abstract}
The growing popularity of Health Social Networking sites has a tremendous impact on people's health related experiences. However, without any quality filtering, there could be a detrimental effect on the users' health. Trust-based techniques have been identified as effective methods to filter the information for recommendation systems. This research focuses on dental care related social networks and recommendation systems. Trust is critical when choosing a dental care provider due to the invasive nature of the treatment. Surprisingly, current dental care recommendation systems do not use trust-based techniques, and most of them are simple reviews and ratings sites. This research aims at improving dental care recommendation systems by proposing a new framework, taking trust into account. It derives trust from both users' social networks and from existing crowdsourced information on dental care. Such a framework could be used for other healthcare recommendation systems where trust is of major importance.
\end{abstract}

\section{Introduction}

The revolution of social media is apparent in the last few years. It has also made a substantial impact on the health care sector and has changed the role of healthcare consumers (both professionals and patients). They have moved from searching information online, to sharing information and interacting with other users (even anonymous) via social media [1]. One of the most popular features of the social media is 'peer reviews' and ratings on any product or service. The specific social networking sites (SNSs) to discuss health issues are referred as Health Social Networks
(HSNs), which includes dental reviews and rating sites. One of the popular HSNs sites, PatientsLikeMe provides valuable health information such as symptoms, clinical diagnosis, treatment options, side effects, sources of medical evidences and opinions about users' experiences of treatments. In addition, HSNs provide opportunities for people to discuss their experiences with other users with similar symptoms and experiences [2]. Moreover, the users can get emotional support by seeing others with similar health symptoms and feel, "I am not alone", which empowers patients and gives them a sense of community, so that they would go back to the site and share more [2]. The process of interaction through HSNs has certainly increased a level of trust to both the website and the user, as an information provider. The interaction could produce and disseminate accurate information faster than traditional method by enhancing collaborations [3, $4,5,6]$. As a result, many dentist reviews and rating sites are emerging, such as DentalCenter, DentalFearCentral, DentistDig, DentistReviewsOnline and $\mathrm{DrOogle}$. Since dental care falls under the category of health, dental professionals have been also listed under the health professionals rating sites such as RateMDs and HealthGrades. In addition, a generic business review site such as Yelp, has been gaining popularity in the US and few other countries for dentist reviews. Amongst all, DrOogle is one of the most dedicated sites for dental professionals in the US, which provides rankings on dentists based on patients' positive reviews [7]. However, the problem is not only there are many different dental reviews and rating sites available but also no consistencies in rating criteria amongst the sites.

Pew Research Center [8] reported a rising number of e-patients and stated that $80 \%$ internet users in the US get health information online, the third most popular online activity after email and search [9]. 
Amongst them, 60\% use social media and 19\% consult rankings of the health care providers. Neiger et al [10] reported that the majority (almost $80 \%$ ) of physicians use one of the social media channels while consulting their patients online.

Due to both pervasive and ubiquitous nature of information and communication technologies (ICTs), the popularity of HSNs has been growing rapidly and the information available through the sites has been changing people's health related experiences [2, 11 , 12]. Nowadays, not all patients are automatically accepting doctors' or dentists' recommendation without doing their own online research [13]. If patients fail to have their needs (e.g. questions, certainty of illness, understanding, etc.) fulfilled through direct communication with doctors or dentists, they may turn to search on the Internet, an alternative source [14, 15]. [16] quoted that a survey indicated $81 \%$ of adult users have used the Internet for health information and acknowledged that the Internet is the most widely used source for health information ahead of doctors, friends and families. But, is the information trustworthy? And is the data accurate? Search engines cannot provide answers to these questions.

There is no doubt that the growth of HSNs offer tremendous opportunities to the healthcare consumers such as, always available, common space for discussing sensitive health concerns, patients empowerment through sharing with other people suffering from similar symptoms and illnesses and medical information in the form of Q/A. [2, 4, 17]. However, these sites do provide some serious challenges: 'how do users know the site, or the information provider is trustable and the provided information is well researched? Are they safe?

Trust has been identified as one of the most important factors for recommendation systems. Trust becomes even more important when it is about health issues due to the possible detrimental effects, resulting in the loss of life. In the context of dental care, due to the invasive nature of treatment and level of dental fear, trust becomes very important.

In this paper, we propose a framework for trustbased dynamic dental care recommendation system, which integrates contextual information in dental care, along with users' relationships and expertise from users' social networks. It also incorporates trust from crowdsouced dental reviews and ratings available.

In section 2 , the proposed framework of trust-based dynamic dental care recommendation system is introduced along with the results from preliminary studies of the users' criteria. The trust evaluation of the framework is elaborated in the sections 3. Finally, the paper is concluded with a discussion.

\section{Proposed framework for dynamic dental care recommendation system}

Trust plays an important role for healthcare consumers to reduce uncertainty in the Internet world [18]. But how do you measure trust in the dynamic environment? We have proposed a trust-based framework for dynamic dental care environment to show how a dental patient can refine the search by measuring trust from the social networks. A framework for dental care recommender system, backed up by profile-based (local) trust and reputation based (global) trust from crowdsourced dental sites can incorporate the concerns people usually have for dental treatments. The proposed framework is shown in the figure 1 .

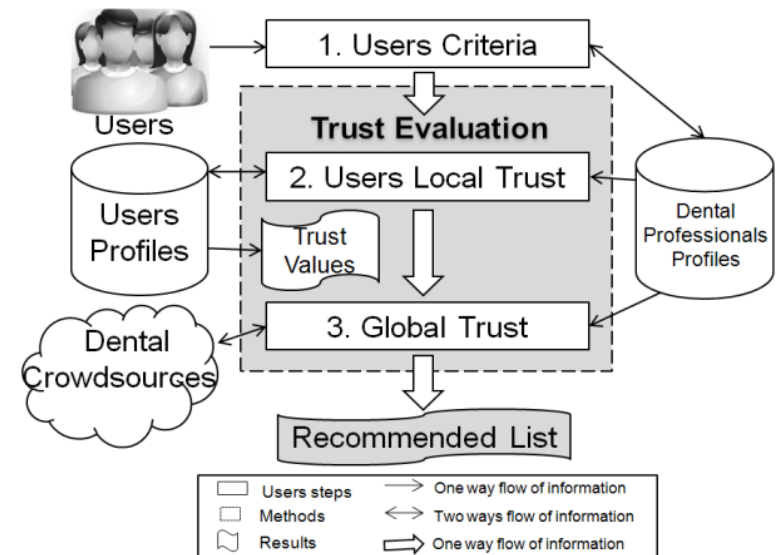

Figure 1: Proposed framework for dynamic dental care recommendation system

At first, the users select their objective and subjective preferences/criteria while searching for the most suitable dental professionals. We have conducted an online survey, and reported initial results on what users prefer while choosing a dentist, in this section. Computation of personalised profile-based trust derived from major components (similarity in dental context, strength of relationships amongst users and expertise in dental care) is the second step. Incorporating trust based on similarities of dental symptoms, hereditary, side-effects and anxiety, strengths of relationhips with other users and their expertise within the network, refines and personalizes the recommendations. Lastly, the recommended list can further be refined by validating with existing crowdsourced dental reviews and rating sites.

[19] showed how additional information of user's criteria provides opportunity for novel multi-criteria recommendation system. In line with this, we have conducted the survey to determine general preference criteria while choosing a dentist. 183 participants completed the survey. The preference criteria would change, depending on the situation the user is, at given 
location and time. Thus, it provides dynamism to the recommendation system.

We prepared a list of criteria which encompasses the criteria required while choosing a dentist. We asked the participants to rate the importance of those criteria in the online survey. Among the criteria, the participants ranked the 'quality of care' and 'quality of service' as the most important criteria while choosing a dentist as shown in the graph in figure 2. Other criteria such as 'reliability', 'location', 'cost', 'reimbursement from insurance', 'waiting time' and 'specialist' are also ranked as very important or important as shown in the figure.

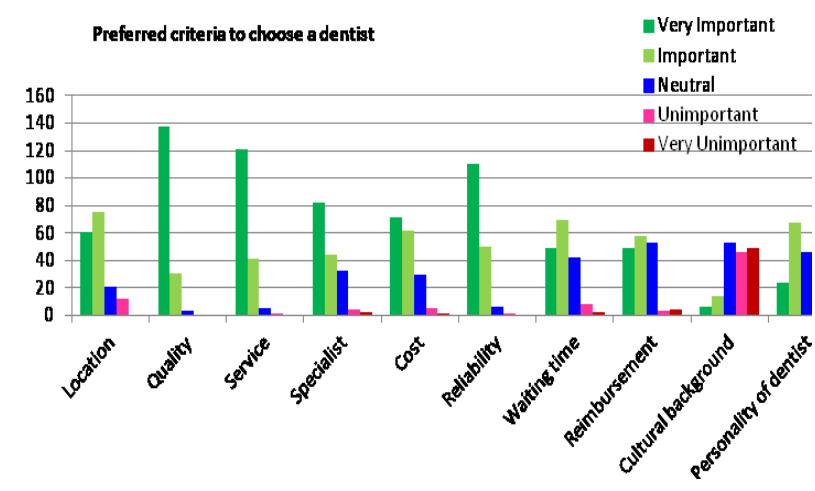

Figure 2: Preferred criteria to choose a dentist ( $y$-axix $=$ number of participants)

Depending on the chosen criteria, the system will recommend a list of dental professionals. How do we know which one from the list to choose from? This research focuses on trust within the users' social network based on their profiles and crowdsrouced dental reviews and ratings, to refine the list as described in the following section.

\section{Trust Evaluation}

Evaluation of trust is done from both local and global aspects. Local trust is derived from the user's own network in dental care. It is evaluated from their profiles and engagements (reviews, ratings, feedbacks, comments or sharing). The global trust is derived from already available crowdsourced online dental reviews and ratings information.

\subsection{Related work}

A study in dental patients [20] revealed that $96.8 \%$ of the participants in Spain (out of total 804 patients) showed some degree of stress about dental treatment. Another dental health survey showed that $46 \%$ of the participants in Australia were anxious about going to the dentist [21]. In an attempt to alleviate and compromise the dental anxiety and give confidence to the patients, dental HSNs are emerging.

The HSNs provides opportunities for information sharing, collaboration and interaction, but there is a chance that users potentially can abuse the system by providing wrong information. Therefore, an appropriate recommendation system is very critical for healthcare information. Trust has been recognised as the most effective factor to determine and filter the right information $[22,23]$. Trust is also identified as a critical factor in the dental care while choosing the best dental professional due to the nature of invasive treatment. Trust-based recommendation systems are able to refine information by utilising personalised profile-based trust within the social networks. Growing popularity in social networks and research on trust within social networks have been main reasons for the increasing trust-based recommendation systems [6]. A person looking for information is referred as 'truster' and the person giving/sharing the information is known as 'trustee' in this context. There are different methods of calculating trust between users. Even the most popular SNS, Facebook (more than a billion users) has now launched Social Graph to recommend and search based on sentiment of users [24]. Summary of the few techniques proposed in the literature are:

TidalTrust [25] used the explicit trust values given by the users of the network to each other. Trust is calculated as a weighted average of the trust values given to trustee by the truster's trusted users. Smyth and O'Donovan [26] defined item-level and profilelevel trust. In general, trust is estimated by measuring past recommendations by the trustee in two levels, general reliability based on profile-level and fine tuned item-level. MoleTrust [27] is very similar to TidalTrust with explicit trust value of the users. However, it looks at the depth-first search by looking at the hop distance from the truster to the trustee because they adopted linear decay in propagating trust through each hop. Matsuo and Yamamoto [28] predicted trust by extracting information from user profiles, reviews and existing trust relations in between users. Skopik et al. [29] used trust relationships between users by looking at the communication between users. Wang et al. [30] estimated trust of users based on similarity in taste (classification of items). They used rating frequency of users to classify the users into different groups of tastes and calculate trust based on common taste. Zhang and $\mathrm{Yu}$ [23] described the category-specific trust relationships between users. In addition, they also used role-based and behavior-based reasoning functions for users' interest and trust relationships. Kim and Phalak [6] measured trust metrics based on users' expertise, preferences and feedback rating data. They believed 
that feedbacks are frequently expressed than explicit trust in the online social network environment. Fernandez-Luque et al [31] defined HealthTrust as a trust for content and member of health community.

\subsection{Users Local Trust (Profile-based)}

In the proposed framework, users local (profilebased) trust is evaluated from major components from user profiles and engagements, such as context similarity, relations and expertise. Similar to what has been considered from some of the literatures related to trust-based recommendation systems mentioned above. Figure 3 shows these components which have significant impact to build trust while searching for trustworthy dental information and professionals.

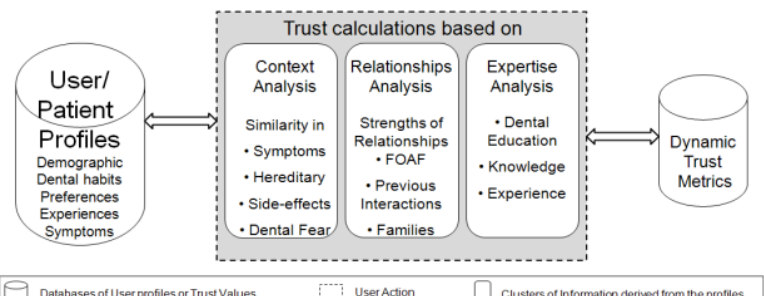

Figure 3: Components of Local Trust

Context Analysis (Similarity): Context is very important while evaluating trustworthiness of a user in the social network. [32] stated "context qualifies a trust opinion, describing what the trusters belief in another's trustworthiness is really about" and extended context into external (situation-specific) and internal (subjectivity) contexts. Context is defined by looking at the similarity information from the user/patient profiles while calculating local trust. In the framework, dental symptoms are basic information that has to be understood well so that truster can look into the information provided by the trustee further. Therefore, similarity of the dental symptoms is the most important factor that can relate to other users in the network. Any other related information such as similarity in dental fear, side effects and hereditary will also strengthen the trustworthiness of the information provider as a trustee.

Relationship Analysis: The strength of the relationship between the users (truster and trustee) within the social network helps analyse trust propagation. The relationship could have already been built due to previous experience in the social network with profile similarity and interaction $[33,34]$, or they knew each other from a long time as a friend or family member, or even a friend of a friend (FOAF). Social networks provide natural environment for users to build trust based on relationships, which relies on connections (and strength of connections) within the social network. It's not only the direct and witnessed experiences but also the social connections through the network are important for trustworthiness [34, 35, 36]. For example, if ' $B$ ' is a friend of your friend ' $A$ ', ' $B$ ' will be more credible, simply based on the relationship. This credibility has some weights while evaluating for trustworthiness of trustee, the information provider. Social network analysis based on the strength of relationships, is another powerful tool to determine the users' trust metric in providing information in the network.

Reputation Analysis (Expertise): A user with the experience and knowledge in the context of dental care is highly regarded (trusted). Truster will trust an expert in the area where s(he) is interested [6] to find specific dental information. Hence, personal knowledge and experience are very important factors, while deriving trust metrics. Users' expertise on dental care can be analysed by number of high quality related information (reviews, feedback or sharing) for specific dental issues and high ratings (reputation) from trusted users in the network [6]. Certainly, this analysis further enhances the trust on the information as well as the trustee in the network.

In summary, personalised profile-based (local) trust within the network can be evaluated from various techniques of the social network analysis (SNAs) in similarity, strength of relationships and expertise. However, measuring subjective attributes for trust calculation, such as similarities, relationships and expertise may not be consistent due to individual perception. Moreover, anonymous users also create a challenge in evaluating trust within the network.

\subsection{Global Trust from Crowdsources}

A significant amount of dental information is publicly available through dental specific and other reviews and rating sites. They all can be categorized as 'crowdsources' for dental information. Some of the emerging dental HSNs have only basic functionalities whereas the others have more, and are gaining popularity. Cross-checking with available information as a global trust as shown in figure 4, is an optional, but useful step in the proposed framework. It is optional because the related information may not be available for particular region.

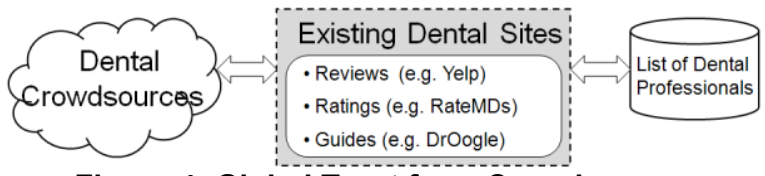

Figure 4: Global Trust from Crowdsources 
There are many sites where the Internet users can search for dentists and get reviews and ratings. The DrOogle site allows dental patients to write reviews and personal feedback to their dentists. Based on positive reviews, the dentists are rated and ranked within a specific location in the US. This site also monitors shilling attacks (biased behavior or making positive reviews to own people) to provide fair ratings to the users [7]. In addition to the reviews/feedback about their dental visits, the patients also can rate the criteria as shown in the table 1. Dental Fear Central is a non-commercial site which provides awareness to the public about the dental anxiety/fear [37]. It is based in the UK, but it has been gradually expanded around the world. This site provides services such as, dental phobia support forum, commons fears in dental and tips to deal with them, psychological ways of tackling dental phobia, tips for dentists and dental FAQ.

Table 1: Criteria used for the dentist ratings

\begin{tabular}{|l|l|}
\hline \multicolumn{1}{|c|}{ Sites } & \multicolumn{1}{|c|}{ Criteria used (1 to 5 Likert Scale) } \\
\hline DrOogle & $\begin{array}{l}\text { 'like a health spa', 'first class service', 'painless } \\
\text { procedures' and 'superb results' }\end{array}$ \\
\hline $\begin{array}{l}\text { National } \\
\text { Dental } \\
\text { Reviews }\end{array}$ & $\begin{array}{l}\text { 'office cleanliness', 'staff cleanliness', 'short } \\
\text { wait time', 'chairside manner' and 'explained } \\
\text { treatment' }\end{array}$ \\
\hline RateMDs & $\begin{array}{l}\text { 'staff friendliness', punctuality', 'helpfulness' } \\
\text { and 'knowledge' }\end{array}$ \\
\hline $\begin{array}{l}\text { Health } \\
\text { Grade }\end{array}$ & $\begin{array}{l}\text { 'scheduling appointments', 'leffice } \\
\text { environment', 'office friendliness', 'wait time', } \\
\text { 'level of trust', 'helps patients understand their } \\
\text { 'time spent with patient' }\end{array}$ \\
\hline
\end{tabular}

Most of the dental crowdsources allow users to find the dentist based on the location and other users' ratings. They also allow the patients to provide free textual feedback/reviews on the dental service. Only in the Health Grade site, there is a short survey questionnaire to measure patient satisfaction. Table 1 shows the criteria used on some of the sites.

Although there is no consistencies on the criteria they use, it is useful to be able to cross-check on the already available information, after evaluating local trust from own social network. However, it has been reported that majority of the ratings and feedback are done by the patients who are either terribly dissatisfied (negative feedback) or very happy with the service (positive feedback). Some people have been raising their voices via blogs and articles about the reviews being flawed. Moreover, the lack of understanding on using reviews sites by which the result could be opposite. For example, a user used one star instead of 5 stars thinking that 1 star is better than multiple stars.

\section{Discussion}

This paper proposes a new trust-based framework for dynamic dental care recommendation system. One main characteristics of the framework is its capacity to verify the information available using profile-based trusted users. It is derived by users' similarity in dental symptoms, side effects and fear, as well as strength of relationships and expertise in the field of dental care. The framework also allows verifying the information from existing crowdsources reviews and ratings sites in dental care. This enables users to refine the overloaded and ignore potentially misleading information.

Thanks to the proposed framework, a user is able to filter the criteria for the search even for unprecedented situations. The system uses the similarity of context and works out the trust metrics to justify the results. Furthermore, it verifies the information with existing crowdsourced dental reviews and ratings sites. The quality of the result varies depending on the location and number of users who has had similar events in dental care in the past and it will improve over time, as more information will be stored in the system.

Social media has been embraced by the dental care industry and the number of dental related reviews and rating sites are increasing [39]. The only way to get the framework presented in this paper implemented is, by getting the different stakeholders involved in dental care onboard. We have been collaborating with users, dentists (MLC Centre Dental Surgery) and regulatory bodies such as Australian Dental Association to identify and understand the key factors for its acceptance, and 'trust' is one of them.

\section{References}

[1] W.B. Lober and J.L. Flowers, "Consumer empowerement in health care amid the internet and social media," Seminars in Oncology Nursing, Vol 27 No 3 (August) 2011 pp. 169-182

[2] M. Swan, "Emerging patient-driven health care models: an examination of health social networks, consumer personalized medicine and quantified self-tracking," International journal of environmental research and public health, Vol 6, No. 2, 2009, pp. 492-525.

[3] M. Swan, "Crowdsourced health research studies: an important emerging complement to clinical trials in the public health research ecosystem," Journal of Medical Internet Research, Vol 14, No.2 2012.

[4] F. Griffiths, J. Cave, F. Boardman, J. Ren, T. Pawlikowska, R. Ball, A. Clarke and A. Cohen ., "Social networks - the future for health care delivery," Social Science \& Medicine, in press, 2012.

[5] L. Weitzel, P. Quaresma and J. P. M. de Oliveira, "Evaluating quality of health information sources," $26^{\text {th }}$ International Conference on Advanced Information Networking and Applications, Fukuoka, Japan, Mar 2629, 2012, pp. 655-662. 
[6] Y. A Kim and R. Phalak, "A trust prediction framework in rating-based experience sharing social networks without a Web of Trust," Information Science, Vol 191, 2012, pp.128-145.

[7] Doctoroogle : http://www.doctoroogle.com

[8] R. Lee, "The rise of the e-patient, understanding social networks and online health information seeking," Pew Internet Project, Pew Research Center, 2012.

[9] L.M. Gallant, C Irizarry, G Boone and G.L. Kreps, "Promoting participatory medicine with social media: New media applications on hospital websites that enhance health education and e-patients voices," Journal of Participatory Medicine, Vol.3 2011

[10] B.L. Neiger, R. Thackeray, S.A. Van Wagenen, C.L. Hanson, J.H. West, M.D. Barnes and M.C. Fagen, "Use of social media in health promotion: Purposes, key performance indicators, and evaluation metrics," Health Promotion Practice, Vol 13, No 2, Mar 2012 pp. 159. 164.

[11] Y.W. Webster, E.R. Dow, J. Koehler, R.C. Gudivada and M.J. Palakal, "Leveraging health social networking communities in translational research," Journal of Biomedical Informatics, Vol 44, 2011 pp. 536-544.

[12] J. Williams, "Social networking applications in health care: threats to the privacy and security of health information," ACM,2010, pp. 39-49.

[13] S.C. Ratzan, "Our new "social" communication age in health," Journal of Health Communication. Vol. 16, No. 8, 2011, pp. 803-4.

[14] J. Hou and M. Shim, "The role of provider-patient communication and trust in online sources in internet use for health-related activities," Journal of Health Communication. Vol. 15, No. S3,2010, pp. 186-99.

[15] N. Tustin, "The role of patient satisfaction in online health information seeking," Journal of Health Communication, Vol. 15, No. 1, 2010, pp. 3-17.

[16] S. Moturu, and H. Liu, "Quantifying the trustworthiness of social media content: content analysis for the social web, LAP Lambert Academic Publishing, 2010.

[17] D. MacLean and S. Hangal, "You didn't tell me that! Visualizing the hidden attributes of online health communities," UIST, Oct 4-7, 2009.

[18] B Hernandez-Orgega, "The role of post-use trustin acceptance of a technology: Drivers and consequences," Technovation, Vol. 31, 2011, pp. 523-538.

[19] G. Adomavicius, N. Manouselis and Y.O. Kwon, "Multi-criteria recommender systems," Recommender Systems Handbook, 2011, pp. 769-803.

[20] L. M. Rodriguez-Vazquez, E. R. Lopez, A. V. Centelles, A.I.B. Otero, F. V. Otero and P. V. Centelles, "Stress amongst primary dental care patients," Med Oral Patol Oral Cir Buscal, April 1, 2008, 13(4), E:253-6.

[21] C. J. Armitage and J.G. Reidy, "Evidence that process simulations reduce anxiety in patients receiving dental treatment: randomized exploratory trial," Anxiety, Stress \& Coping: An International Journal, 25:2, 2011, pp. 155-165.

[22] D. O'Doherty, S. Jouili and P. Van Roy, "Trust-based recommendation: an empirical analysis," The $6^{\text {th }}$ SNA_KDD Workshop, Aug 12, 2012, Beijing, China.

[23] Y. Zhang and T. Yu, "Mining trust relationships from online social networks," Journal of computer science and technology, Vol 27, No. 3, May 2012, pp. 492-505.

[24] L. Ulanoff, " Facebook graph search could be its greatest innovation," Mashable.com, 16 Jan 2013, available

http://mashable.com/2013/01/15/facebook-graphsearch-great/

[25] J.A. Golbeck, "Computing and applying trust in webbased social networks," PhD Thesis, University of Maryland, 2005

[26] B. Smyth and J. O'Donovan, "Trust in recommender systems," $10^{\text {th }}$ international conference on intelligent user interfaces, 2005, pp. 167-174.

[27] P. Avesani, P. Massa and R. Tiella, "A trust-enhanced recommender system application: Moleskiing," ACM Symposium on Applied Computing, 2005

[28] Y. Matsuo and H. Yamamoto, "Measuring bidirectional effects on trust and rating on online social networks," The $18^{\text {th }}$ International Conference on World Wide Web, Madrid, Spain, April 20-24, 2009, pp. 751.760.

[29] F. Sokpik, H. Truong and S. Dustdar, "Trust and reputation mining in professional virtual communities," The $9^{\text {th }}$ International Conference on Web engineering, San Sebastian, Spain, June 22-26, 2009, pp. 751-760.

[30] J. Wang, J. Yin, Y Liu and C. Huang, "Trust-based collaborative filtering," The $8^{\text {th }}$ International Conference on Fuzzy Systems and Knowledge Discovery, Shanghai, China, July 26-28, 2011, pp. 2650-2654.

[31] L. Fernandez-Luque, R. Karlsen and G. B. Melton, "HealthTrust: a social network approach for retrieving online health videos," Journal of Medical Internet Reseach, Vol. 14 No. 1, e 22, 2012.

[32] M. Tavakolifard and K.C. Almeroth, "Trust 2.0: who to believe in the flood of online data?" International Conference on Computing, Networking and Communicatinos, 2012.

[33] R. Xiang, J. Neville and M. Rogati, "Modeling relationship strength in online social networks," The International World Wide Web Conference, Raleigh, North Carolina, USA, Apr 26-30, 2010, pp. 981-990.

[34] M. Kwan andD. Ramachandran, "Trust and online reputation systems," Computing with Social Trust, 2009, pp. 287-311.

[35] Y.A. Kim, M.E. Eisenbergb, M.A. Ahmada, J. Srivastavaa, "Modeling Trust in Online Social Networks to Improve Adolescent Health Behaviors," (2011).

[36] V. Nguyen, E. Lim, J. Jian and A. Sun, "To trust or not to trust? Predicting online trusts using trust antecedent framework," $9^{\text {th }}$ IEEE International Conference on Data Mining, 2009, pp. 896-901.

[37] DentalFearCentral: http://www.dentalfearcentral.org

[38] X. Su, and T.M. Khoshgoftaar, "A Survey of Collaborative Filtering Techniques," Hindawi Publishing. Advances in Artificial Intelligence. 2009.

[39] M.K.K. Devi and P. Venkatesh, " Smoothing approach to alleviate the meager rating problem in collaborative recommender systems," Future Generation Computer Systems, Vol 29, 2013, pp. 262-270. 